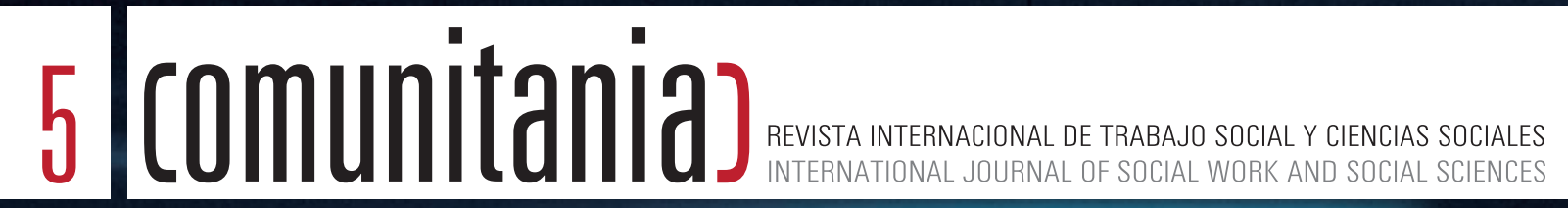

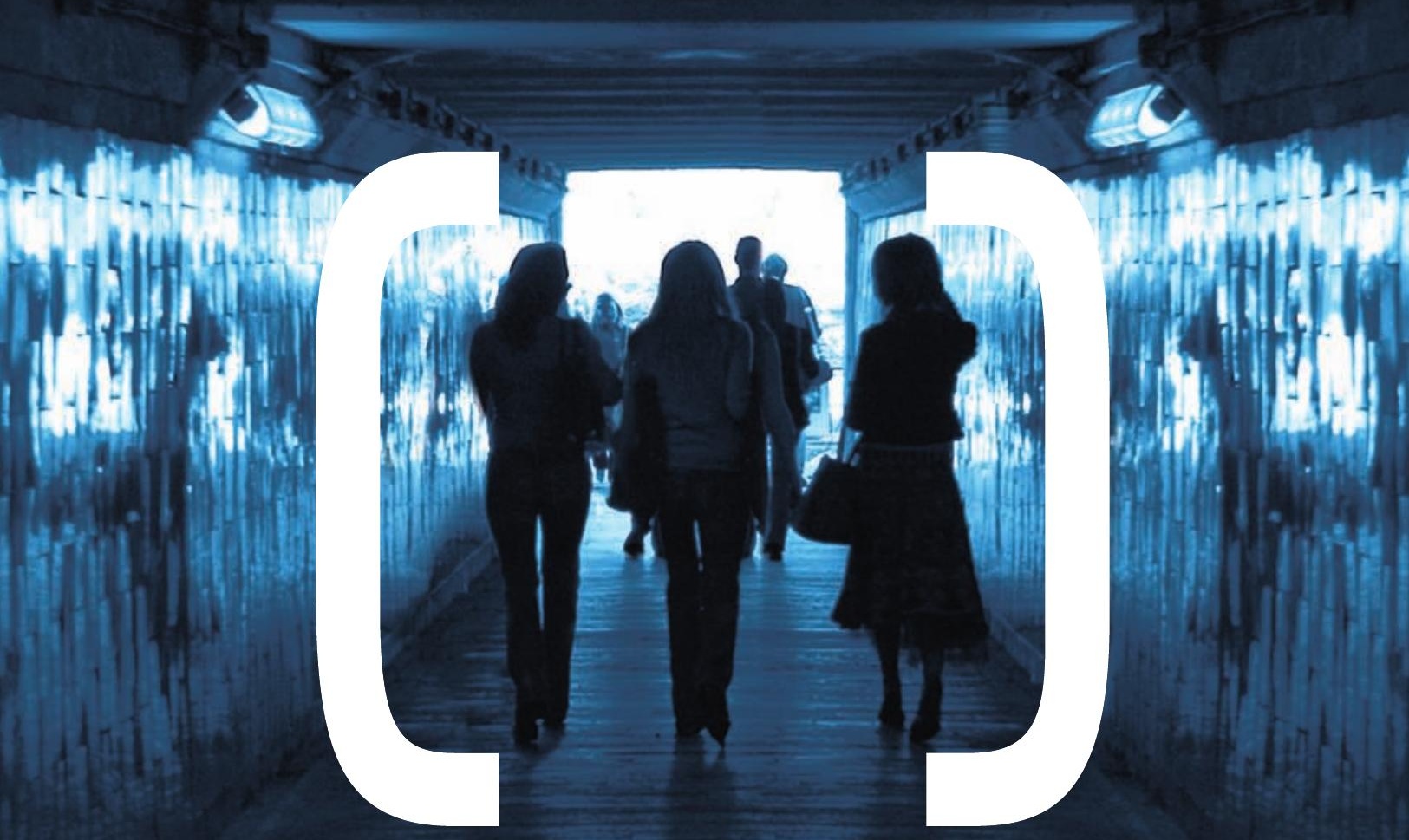

EGUZKI URTEAGA | RODRIGO ANDRÉS | ÁNGELES MARTíNEZ | Ma INMACULADA LÓPEZ | ALEJANDRO CABELLO MARÍA PAZ GARCÍA-LONGORIA | REYNA LIZETH VÁZZUEZ | ARNULFO SÁNCHEZ | EVA SOTOMAYOR | YOLANDA DE LA FUENTE MARTA GARCÍA | Ma LUISA GRANDE | TOMÁS ALBERICH | KARLA SÁENZ | DELIA TÉLLEZ | ANTONIO GUTIÉRREZZ 


\title{
Estado de bienestar e inversión social en Europa Welfare State and Social Invesment in Europe
}

\author{
Eguzki Urteaga* \\ * Universidad del País Vasco. eguzki.urteaga@ehu.es
}

\begin{abstract}
Abstrac:
During the seventies, the economic crisis has questioned the relevancy of the Keynesian policies of reactivation and, in front of the increase of the unemployment, the model of Welfare state restored from 1945 has been widely criticized by the neoclassic economists who were defending offer politics that suppose a reduction of the wage costs and social protection. But, the balance of the neoliberal policies is disappointing to socioeconomic level because, beside not guaranteeing a strong and constant growth, it has extended the social inequalities. If the traditional systems of protection are not totally adapted to the economic and social current context, a new approach of the social policies appears from the beginning of the 2000s that insists on the reorientation of the Welfare states. It is a question of intervening as soon as possible to correct the inequalities and of anticipating the situations of professional exclusion. It is a question equally to favor the gender equality in labor market and to reorganize his careers. This strategy of social investment answers also to the requirements of strong qualification of the post-industrial economy.
\end{abstract}

Keywords: Welfare state - reform - social politics - social investment - social cohesion.

\section{Resumen:}

Durante los años setenta, la crisis económica ha cuestionado la pertinencia de las políticas de reactivación keynesianas y, ante el incremento del desempleo, el modelo del Estado de bienestar instaurado a partir de 1945 ha sido ampliamente criticado por los economistas neoclásicos que defendían una política de la oferta que pasaba por una reducción de los costes salariales y de la prestación social. No en vano, el balance de las políticas neoliberales es decepcionante a nivel socioeconómico ya que, además de no garantizar un crecimiento notable y continuo, ha ampliado las desigualdades sociales. Si los sistemas tradicionales de protección no se adecuan plenamente al contexto económico y social actual, un nuevo enfoque de las políticas sociales aparece a partir del inicio de los años 2000 que insiste en la reorientación de los Estados de bienestar. Se trata de intervenir lo antes posible para corregir las desigualdades y prevenir las situaciones de exclusión profesional. Es cuestión igualmente de favorecer la igualdad de género en el mundo laboral y de reorganizar sus trayectorias y carreras. Esta estrategia de inversión social responde también a las exigencias de fuerte cualificación de la economía posindustrial. 
Palabras clave: Estado de bienestar - reforma - política social - inversión social - cohesión social.

\section{Article info:}

Received: 20/03/2012 / Received in revised form: 31/08/2012

Aceppted: 29/12/2012 / Published online: 20/02/2013

DOI: http://dx.doi.org/10.5944/comunitania.5.1

\section{Introducción}

La evolución de las concepciones del Estado de bienestar, su rol y sus misiones, debe ser relacionada con las evoluciones del capitalismo. Nacidos con la revolución industrial, los sistemas de protección social se han desarrollado en congruencia con la industria y el consumo de masas. Mientras que el modelo económico de losTreinta Gloriosos (Fourastié, 1979) es fuertemente cuestionado a partir de la mitad de los años setenta en beneficio del capitalismo financiero, la protección social está acusada de ser una de las causas de la crisis y su adaptación al contexto internacional es preconizada con el fin de que sea menos costosa y más favorable para el empleo. Desde el final de los años noventa, esta visión negativa de la protección social deja lugar progresivamente a un nuevo enfoque (Esping-Andersen, 2002; Esping-Andersen y Palier, 2008) que intenta a la vez responder a las nuevas necesidades sociales y acompañar la mutación del capitalismo hacia una economía del conocimiento basada en la innovación (Aghion, 2010; Aghion et al., 2010) y los servicios (Herzog, 2004).

\section{El modelo keynesiano}

Los sistemas de seguro social, figura principal del Estado de bienestar en Europa continental, son la emanación y el soporte de la sociedad industrial. Nacen en el siglo XIX con la revolución industrial y su correlato social: la aparición del asalariado ${ }^{1}$ (Castel, 1999). Destinados a garantizar la continuidad de la renta de los obreros que han perdido las solidaridades familiares y locales de la sociedad agrícola, permiten simultáneamente a los empresarios asegurarse su fidelidad, la estabilidad y la calidad de su mano de obra.

\footnotetext{
1 Los trabajadores se benefician de protecciones legales y reglamentarias cada vez más amplias y se reconoce el rol de los sindicatos. Las políticas económicas y las políticas salariales encuentran su complemento natural en las políticas de protección social. Esta se extiende progresivamente a todas las categorías de la población, asalariadas y posteriormente no asalariadas (Flora, 1986). Los seguros sociales conceden prestaciones cada vez más generosas, dando una legitimidad creciente a la Seguridad social. La idea de solidaridad legitima el sistema de los flujos financieros y de los flujos de prestaciones.
} 
A lo largo de los treinta años posteriores a la Segunda Guerra Mundial, el fordismo y los enfoques keynesianos de las políticas económicas permitirán un verdadero incremento del gasto social, ya que pasa del 5\% al 25\% del PIB entre 1945 y 1979 para el conjunto de los países de Europa occidental. Durante ese periodo, las políticas sociales y económicas parecen reforzarse mutuamente ${ }^{2}$. Los dispositivos de protección social permiten apoyar y reactivar el crecimiento económico: son creadores de empleo; mantienen una capacidad de consumo para aquellos que no pueden trabajar; en la medida en que garantizan una renta, liberan el ahorro de protección y permiten dedicar una parte creciente de la renta al consumo; son igualmente unos instrumentos de reactivación económica, a través de un aumento de las prestaciones sociales o de las creaciones por empleo en los servicios públicos ${ }^{3}$. El crecimiento económico de los Treinta Gloriosos (1945-1975) se fundamenta, en gran parte, en las interacciones virtuosas entre el desarrollo de la industria de producción de bienes estandarizados de gran difusión, el consumo de masas y la protección social (Flora, 1986).

\section{La crítica neoliberal}

Con la crisis que se inicia en la mitad de los años setenta y el fracaso de las políticas keynesianas de reactivación, el Estado de bienestar entra en una nueva fase. A lo largo de los años ochenta, se convierte en el punto de mira de las críticas de los economistas neoclásicos (Von Mises, 1975; Hayek, 1998) relevados por los líderes políticos neoliberales. Estos subrayan la necesidad de orientar los esfuerzos, no tanto hacia la demanda (los consumidores) sino hacia la oferta (las empresas y los inversores) reduciendo los costes asumidos por las empresas, especialmente el coste laboral que se incrementaría con las cotizaciones sociales, las rigideces del mercado laboral que impedirían las contrataciones, y las prestaciones sociales demasiado generosas e incondicionales que desanimarían las personas a buscar un empleo.

2 La noción de economía mixta es la palabra clave de esa época (Shonfield, 1967), dado que lo social está considerado como el complemento indispensable de lo económico. El mercado es percibido de manera negativa (markets failures como consecuencia de las externalidades negativas o de la competencia imperfecta), mientras que la capacidad de planificación y de intervención del Estado está asociada a ciertas virtudes.

3 Las décadas de la posguerra pueden considerarse retrospectivamente como un "edad de oro" de los Estados de bienestar. Bajo la égida del régimen internacional de Bretton-Woods (1944), los Estados están legítimamente autorizados a constituir unos sistemas económicos productivos y sociales nacionales que escapan parcialmente a las evoluciones erráticas de los mercados. Los países controlan su economía y su moneda y pueden llevar a cabo a su manera las políticas de modernización. La intervención del Estado en la economía es fuerte y los sectores económicos protegidos son numerosos. El sistema de paridades fijas permite los reajustes necesarios mientras que el keynesianismo se convierte en la teoría que justifica las opciones económicas notablemente antiliberales y pro-intervencionistas de los modernizadores. 
Durante los años ochenta y noventa, numerosas reformas intentarán adaptar los sistemas de protección social a estos nuevos planteamientos económicos dominantes, especialmente por unas políticas de reducción del nivel de las prestaciones sociales, de privatización de ciertos seguros sociales y de condicionamiento de las prestaciones concedidas a las personas inactivas (Urteaga, 2009a), con el fin de incentivar la búsqueda de empleo. Esta adaptación de los sistemas de protección social a una política de oferta aspira a poner el Estado de bienestar al servicio de la competitividad de las empresas, de los Estados y de los individuos. Otro principio general, congruente con la necesidad de controlar el gasto, consiste en el hecho de centrar la intervención pública en aquellos que tienen la mayor necesidad en lugar de promover unas políticas sociales universales. Se trata, por último, de hacer un llamamiento a todos los actores de la protección social: Estado, mercado, familia y sector asociativo para promover una protección social que sea más eficaz y próxima de los individuos que aquella concedida por la administración.

Las reformas estructurales de la protección social llevadas a cabo durante los años ochenta en Reino Unido, los años noventa en los países nórdicos (Kautto et al., 1999; Urteaga, 2007) y los años 2000 en los países de Europa continental (Urteaga, 2009b), siguen una orientación similar. Si los caminos para alcanzar ese objetivo son muy diferentes, la activación de las políticas de empleo aparece como un objetivo general. El aumento de las tasas de actividad se ha convertido en un principio de acción común para enfrentarse al desempleo y al problema de las pensiones de jubilación $n^{4}$. En el ámbito de las pensiones, la mayoría de los países desarrollan un sistema que se apoya en varios pilares (Ramos y Del Pino, 2009) que incluye los principios del reparto y de la capitalización, y ponen el énfasis en la importancia de la "contributividad" (vínculo entre el nivel de la pensión y el volumen de las cotizaciones pagadas). Los cambios son especialmente importantes para los sistemas basados en el seguro y el reparto, a la imagen de los países de Europa continental. En el sector sanitario ${ }^{5}$, la competencia

${ }^{4}$ En materia de pensiones de jubilación, la preocupación concierne el coste de las mismas, lo que ha provocado unas reformas de "segunda generación" (Gillion, Turner, Bauley y Latulippe (2000), a veces paramétricas y a veces más radicales, que, en ambos casos, se traducen por una reducción del nivel de las pensiones. En los hechos, las reformas de los últimos quince años se caracterizan por unos puntos comunes: toma en consideración superior de las contribuciones en el cálculo del importe de la pensión, alargamiento de la vida activa, primacía de las cotizaciones, orientación hacia un modelo mixto basado en tres pilares (universal, profesional obligatoria o no, individual). Los Estados de bienestar de tipo bismarckiano han utilizado dos estrategias complementarias: una presión directa que pasa por el retraso de la edad de obtención de la jubilación completa y una presión indirecta para el aumento del número de contribuciones en el marco del régimen por repartición. Esto conduce a una mayor individualización y a una menor solidaridad mutua en el marco del seguro de vejez. Estas reformas tendrán como consecuencia una disminución, a menudo sustancial, de la tasa de sustitución de las pensiones. Los estudios de la OCDE (2008) muestran una bajada muy significativa del orden del $40 \%$ en Portugal, del $27 \%$ en Alemania y del $20 \%$ en Suecia.

${ }^{5}$ La necesidad de controlar el gasto y de mejorar la protección de las personas ante los nuevos riesgos ocupa el primer lugar en la agenda política. Un consenso se impone en la mayoría de los países sobre la necesidad de reformar unos sistemas considerados como demasiado costosos y/o insuficientemente 
se ha incrementado, entre los que ofrecen atención en los sistemas nacionales de sanidad y entre los aseguradores en los sistemas de seguro de enfermedad. Actualmente, todos los países intentan favorecer el empleo femenino, sobre todo a través del desarrollo de las políticas de atención de la dependencia (Pierson, 2001; Palier, 2010; Urteaga, 2012).

Si se hace el balance, todas estas políticas no han permitido reducir el nivel global del gasto social pero lo han estabilizado, a pesar de que la demanda social se haya incrementado notablemente, lo que supone una bajada de las prestaciones sociales individuales. Tampoco han permitido estimular un fuerte crecimiento económico, al menos en Europa continental, y cuando han permitido crear empleo, son a menudo unos empleos de baja calidad, atípicos, precarios y mal remunerados. Se han traducido por un incremento notable de la pobreza y de las desigualdades (OCDE, 2008).

\section{El Estado de bienestar ante la globalización}

A pesar de que las reformas intentaban cambiar los sistemas de protección social, las economías y las sociedades de los países occidentales evolucionaban rápidamente. La globalización de los intercambios y la circulación de los capitales han desplazado las actividades económicas, deslocalizando hacia los países de Europa del Este y sobre todo hacia Asia las actividades industriales de masas que gozan de una mano de obra barata y poco cualificada ${ }^{6}$. Esta evolución conduce los países antigua-

eficaces. Los sistemas nacionales de sanidad introducen una "competencia regulada". En los sistemas bismarckianos de seguro social, las reformas aspiran a encauzar el nivel de gasto así como a aumentar y a diversificar los ingresos (Palier, 2010). Aunque limitadas, estas reformas hacen correr el riesgo de disminuir la equidad en materia de atención sanitaria. Conviene observar igualmente que numerosos países toman medidas para asegurar el acceso a la atención médica de las personas más desfavorecidas no cubiertas por los sistemas clásicos de protección social vinculadas al empleo. Además, todos los países industrializados están concernidos por el coste creciente de la atención médica de larga duración vinculada al incremento de las personas mayores dependientes. Algunos países ya han puesto en marcha nuevos sistemas mientras que otros están a la búsqueda de una atención adecuada.

${ }^{6}$ La globalización produce una competencia internacional exacerbada que penaliza los países con un alto nivel de salario real. La internacionalización de los intercambios reduce a la vez los costes de los intercambios y aumenta los beneficios asociados. La disminución del coste de los transportes y de la comunicación permite la entrada en la competencia económica de países con niveles salariales bajos capaces de producir a menor coste los objetos manufacturados que exigen una fuerte intensidad de trabajo. Para no conocer un incremento rápido del desempleo y de la exclusión, los países industrializados tienden a proceder a la bajada generalizada de los costes laborales. Resulta de todo ello una descalificación relativa de los trabajadores de los países del Norte y, por lo tanto, la marginación más o menos rápida de una fracción de la fuerza de trabajo (Wood, 1994). Para mantener sus beneficios, las empresas intentan racionalizar sus actividades productivas, limitar los costes fiscales y sociales que pesan sobre su competitividad, e implantar sus actividades productivas en los países con bajas tasas de salario y de protección social. Barry Bluestone y Bennett Harrison (1982) han sido los primeros en poner de manifiesto los efec- 
mente industrializados a reconvertirse en nuevas actividades posindustriales (EspingAndersen, 1996; 1999) basadas a la vez en la innovación tecnológica, la alta cualificación, el saber y los servicios, especialmente los servicios a la persona (Cohen, 2006). Las protecciones sociales basadas en los seguros sociales, concebidas originariamente para proteger a los obreros industriales poco cualificados que tenían un contrato indefinido, a menudo en los sectores industriales o de servicios clásicos, resultan poco adecuadas para proteger unas trayectorias profesionales más móviles, caóticas y precarias, típicas de la nueva economía. Los trabajadores asalariados protegidos se hallan en una situación más precaria, como consecuencia tanto de las evoluciones demográficas como de las mutaciones económicas que corren el riesgo de debilitar unas protecciones que estaban anteriormente bien establecidas.

Si la economía se ha transformado, las sociedades han cambiado igualmente a lo largo de estos últimos veinte años, dado que ciertas mutaciones sociales tienen un impacto directo sobre los Estados de bienestar. El envejecimiento de la población (Blanpain y Chardon, 2010), debido a la vez al alargamiento de la duración de la vida y a la reducción del número de nacimientos, es un hecho fundamental que pesa sobre el equilibrio futuro de los sistemas de protección social. Estos sistemas están igualmente trastornados por las mutaciones de las estructuras familiares. A lo largo de las últimas décadas, el formato dominante de la familia en el cual el marido trabaja y la mujer se queda en casa ha sido cuestionado por la diversificación de los modelos familiares y, especialmente, las familias mono-parentales. Los sistemas de protección social han sido concebidos, lo más a menudo, para proteger a los trabajadores y a sus familias. Dan acceso a las protecciones a través de los derechos sociales del marido que trabaja y, por lo tanto, no están capacitados para ofrecer una protección adecuada a las mujeres-madres solteras, ni a los jóvenes desempleados, ni a los desempleados de larga duración.

tos negativos de la nueva división internacional del trabajo. Según su análisis, las multinacionales han decidido deliberadamente deslocalizar sus inversiones industriales en los países en vía de desarrollado que practican unas políticas salariales restrictivas con el fin de restaurar sus niveles de rentabilidad. Esta estrategia marca, por lo tanto, la ruptura de un pacto implícito entre el trabajo, el capital y el Estado (Keohane y Milner, 1996; Garrett, 1998; Manov, 2001), conduciendo a una crisis de desindustrialización y a un cuestionamiento de los acuerdos sociales de la posguerra.

Además, la nueva economía globalizada se traduce por el debilitamiento rápido de la autonomía de las políticas nacionales (Sassen, 1996). La notable apertura económica genera en todos los países una gran vulnerabilidad ante los movimientos internacionales de capital y, poco a poco, sus márgenes de maniobra se reducen. El final del sistema de paridades fijas establecido por los acuerdos de Bretton Woods y la bajada generalizada de las barreras arancelarias y reglamentarias impulsada por el GATT (General Agreement on Tariffs and Trade) y posteriormente por la OMC (Organización Mundial del Comercio) convierten los Estados en muy vulnerables ante los movimientos de capital a corto plazo. Ya no es posible poner en marcha unas políticas económicas keynesianas expansionistas, sinónimas de déficit presupuestario elevado y de riesgo inflacionista, sin correr el riesgo de una rápida sanción por los mercados internacionales de capital. Los países ya no disponen de la libertad de llevar a cabo unas políticas socioeconómicas totalmente autónomas. 
Los sistemas de protección social tradicionales, basados en los seguros y las transferencias sociales, resultan igualmente incapaces de acompañar el movimiento de fondo que ha conducido un número creciente de mujeres a trabajar. Para ello, estas, además de reconocer los derechos sociales, deben poder beneficiarse de servicios sociales (guarderías, atención a domicilio para las personas mayores o discapacitadas); los cuales no están suficientemente desarrollados. Si los debates se focalizan en las nuevas necesidades generadas por el envejecimiento de la población, se olvida, sin embargo, que la pobreza se ha desplazado. Ya no se concentra en las personas mayores, sino en los jóvenes, las mujeres que viven solas con sus hijos, las personas sin cualificación y los desempleados de larga duración. Todas estas personas están poco protegidas por los sistemas tradicionales que se han centrado en las pensiones y en la sanidad que conciernen sobre todo a las personas mayores.

Ante estas evoluciones, una renovación del Estado de bienestar es indispensable con el fin de acompañar las mutaciones de la economía y de la sociedad, y preparar el futuro.

\section{Apostar por la inversión social}

Desde el inicio de los años 2000, una nueva perspectiva de las políticas sociales ha aparecido que defiende la reorientación del Estado de bienestar hacia el futuro (Esping-Andersen, 2002; Esping Andersen y Palier, 2010). Inicialmente destinadas a compensar la pérdida temporal o definitiva de la renta de los trabajadores, las políticas sociales intervienen lo más a menudo una vez que el riesgo se ha concretado. Las ayudas sociales aspiran a apoyar a los que se encuentran en dificultad, una vez que se han convertido en pobres. La indemnización del desempleo, las políticas de inserción y de retorno al mercado laboral intervienen con posterioridad, después de que la persona haya sido despedida o excluida del mercado laboral. Asimismo, el gasto sanitario financia a menudo la curación en lugar de pagar la prevención. La parte más importante del gasto social, la que está dedicada a las pensiones de jubilación, acontece al final de la vida, una vez las carreras profesionales llevadas a cabo, y sanciona los que tienen una trayectoria atípica (sucesión de contratos precarios, interrupción de carreras, jornada parcial). Se trata de preparar en lugar de arreglar, de prevenir, de apoyar y de dotar a los individuos y no de dejar jugar el mercado y, posteriormente, de indemnizar a los perdedores.

Esta perspectiva concibe estos nuevos gastos sociales no como un coste para la economía sino como una serie de inversiones, necesarias a la vez para garantizar un crecimiento duradero, fuerte y compartido, y para responder a las nuevas necesidades sociales. La estrategia de inversión social define unas prioridades inéditas para el Estado de bienestar con el fin de acompañar a los individuos a lo largo de sus trayectorias vitales. Se trata de mejorar la ayuda prestada a las mujeres, los jóvenes y 
los menores. Es cuestión igualmente de reorganizar el trabajo y de transformar las carreras profesionales.

\subsection{Prioridad a las mujeres y los menores}

Mientras que los sistemas actuales de protección social generan un gasto creciente para las personas mayores, la estrategia de inversión social intenta priorizar a las mujeres y a los menores. Subraya hasta qué punto es necesario invertir en estos colectivos. En lugar de luchar contra las situaciones de exclusión social y de formar de nuevo a la mano de obra, es preferible concentrar los esfuerzos en una óptica preventiva centrada en la infancia. Luchar contra la pobreza infantil y garantizarles unas mejores condiciones de cuidado y de desarrollo debe permitir simultáneamente prevenir la exclusión (la pobreza es superior entre los adultos provenientes de familias pobres) y preparar una mano de obra mejor formada, cualificada y móvil (las dificultades escolares pueden ser reducidas por una socialización precoz en las guarderías).

1. Crear un servicio público de la pequeña infancia. Para ello, es necesario a la vez garantizar una renta mínima (Urteaga, 2011) a todas las familias (no abandonar las antiguas políticas redistributivas e incluso desarrollarlas, dado que la lucha contra los efectos de la pobreza y de la precariedad familiar sigue siendo fundamental) y favorecer el desarrollo de los modos colectivos de atención de los niños que garantice una buena socialización primaria y unas condiciones de aprendizaje capaces de preparar convenientemente el futuro. Hoy en día, el desempleo afecta sobre todo a los menos cualificados y a las personas cuyas cualificaciones son obsoletas.

Muchas cosas se juegan durante los primeros años. Las capacidades cognitivas, comunicativas y relacionales necesarias para el éxito escolar y profesional se adquieren antes incluso de alcanzar la edad de la escolarización obligatoria. Los niños nacidos en los entornos favorecidos se benefician de numerosas oportunidades de desarrollar sus capacidades, lo que no sucede en los entornos desfavorecidos. Dar una oportunidad a todos desde la infancia pasa por un servicio público de la pequeña infancia (Urteaga, 2009c). Semejantes servicios expresan el espíritu de este nuevo modelo de protección social de dos maneras: invierten en el capital humano del niño procurándole a una edad precoz una atención y una educación estimulantes; e invierten en el capital humano de la madre permitiéndole permanecer en el mercado laboral.

Desarrollar unas estrategias de acogida de calidad para los menores permite, no solamente prepararlos a su futura vida sino también responder a las necesidades de las mujeres y de las familias. Compatibilizando vida familiar y vida profesional, estas estructuras favorecen el empleo de las mujeres. Esto corresponde a la voluntad de estos últimos de adquirir una autonomía financiera con respecto a los hombres, pero 
también a una doble necesidad social: reducir los riesgos de pobreza de los niños (la pobreza es siempre menor en las familias donde los padres trabajan) y aumentar las tasas generales de empleo. Desarrollar unas estructuras de acogida de calidad para los más jóvenes permite además crear en ese sector unos empleos estables, cualificados y protegidos, al contrario de los empleos privados de servicios domiciliarios que son a menudo a jornada parcial, poco cualificados, mal remunerados y precarios.

2. Favorecer la igualdad de género en el trabajo. Favorecer el trabajo de todos en las mejores condiciones supone plantear la cuestión de la igualdad de género en el ámbito profesional. Mientras que las jóvenes mujeres consiguen mejores resultados escolares y universitarios, sus carreras profesionales son peores. Están peor remuneradas, trabajan más a menudo a jornada parcial y conocen una menor progresión en la jerarquía de las responsabilidades. Todo ello se traduce por unas pensiones inferiores. Las mujeres deben pagar el precio profesional porque interrumpen 0 reducen su carrera para poder cuidar de sus hijos y, posteriormente, de las personas mayores dependientes. Favorecer una mayor igualdad de género permite a más mujeres tener las carreras que desean, correspondientes a sus cualificaciones, lo que exige una política de igualdad en la esfera familiar, una profunda restructuración de los permisos parentales con el fin de incentivar las familias a compartir la carga de los niños (instauración de un permiso parental más corto pero mejor remunerado y que se beneficia de una bonificación si es compartido por los padres).

Repensar el sistema educativo. Permitir a todos tener un buen empleo implica repensar el funcionamiento del sistema educativo. La estrategia de inversión social implica no solamente un incremento de los presupuestos dedicados a la educación sino también una reconsideración de la enseñanza y de su organización para que todos puedan ir lo más lejos posible en sus estudios (evitar las repeticiones de curso, favorecer la diversidad social en las aulas y centros, y las materias troncales, multiplicar el seguimiento en pequeños grupos, etc.). A menudo no se concibe la política educativa como un componente de las políticas sociales. Pero, se espera del sistema educativo que juegue un rol de movilidad social que disfunciona hoy en día. Las comparaciones internacionales muestran que tres elementos explican la reproducción masiva de las desigualdades sociales a través del sistema educativo: 1) la importancia de la experiencia preescolar, 2) la escasa inversión en la enseñanza preescolar, primaria y universitaria, y 3) la selección de los mejores en lugar de buscar la cualificación de todos (Baudelot y Establet, 2009).

\subsection{Reorganizar el trabajo y las trayectorias profesionales}

Denominada nueva economía, economía del conocimiento, economía inmaterial o capitalismo cognitivo, la economía posindustrial no ha encontrado todavía su denominación. No en vano, su principal motor es la innovación, el saber, la información y la inteligencia. Por lo tanto, es importante no solamente para los individuos sino tam- 
bién para el crecimiento económico (Aghion y Cohen, 2004) invertir en lo que permitirá aumentar el nivel general de cualificación de los trabajadores, es decir de la pequeña infancia y el sistema educativo, así como la formación profesional a lo largo de la vida. El mundo laboral se ha transformado con la nueva economía.

Las nuevas tecnologías de la información y de la comunicación han acelerado el ritmo del cambio. Cualquier conocimiento o habilidad se convierte en rápidamente obsoleto. En ese contexto, la clave del éxito económico es la capacidad de renovar constantemente las actividades más expuestas a la competencia mundial. Transformar las empresas en organizaciones que saben aprender y cambiar, y permitir a los asalariados inscribirse en un proceso de renovación constante de las competencias se convierte en algo fundamental.

Mientras que la estrategia de competitividad basada en la reducción de los costes, la productividad y la gestión por el estrés de los trabajadores muestra sus límites, se descubre que las economías más dinámicas y más innovadoras de Europa son aquellas que han sabido mejorar las condiciones de trabajo para todos, reducir las diferencias salariales, favorecer el trabajo creativo y la autonomía, y desarrollar las lógicas de aprendizaje permanente en el seno de la empresa. Con el fin de evitar la polarización del mercado laboral entre aquellos cuyas competencias se incrementan y se renuevan y aquellos cuyas cualificaciones no cambian, e incluso se deterioran al final de su carrera, es fundamental desarrollar una política de formación profesional para todos y a lo largo de toda la vida. Cada año, cerca de un tercio de los trabajadores están en formación profesional en Dinamarca o en Suecia frente al $7 \%$ en Francia.

Entre las políticas que favorecen el retorno al empleo de los desempleados, ciertos países ponen el énfasis en la colocación y la reinserción laboral a toda costa, incluso para ejercer un empleo que exige un nivel de cualificación inferior, mientras que otros prefieren subvencionar empleos poco cualificados, especialmente a través de la exoneración de cargas sociales y los complementos retributivos a los trabajadores pobres. Apostando por los empleos de calidad, la estrategia de inversión social se basa en la organización de las transiciones profesionales, el acceso de todos a unas formaciones largas y cualificantes a lo largo de la vida, y el acompañamiento de las movilidades por una garantía de renta elevada en periodo de formación profesional y de búsqueda de empleo, sin pérdida de derechos sociales. En estas condiciones, la movilidad profesional o el alargamiento de la duración de las carreras acaban siendo socialmente aceptables.

\section{Conclusión}

Desde hace una década, los debates internacionales sobre el futuro del Estado de bienestar (Merrien, Parchet y Kernen, 2005; Del Pino, 2009) plantean la cuestión de 
utilizar de otra forma los fondos sociales con el fin de responder a la vez a las nuevas necesidades y de preparar el futuro. Modificación de los permisos parentales, fortalecimiento del servicio público de la pequeña infancia, promoción escolar para todos, inversión en la educación y la investigación, creación de empleos de calidad en los servicios a la persona, mejora de las condiciones de trabajo, reducción de las disparidades salariales, autonomía y aprendizaje permanente en las empresas, formación y recualificación profesional de los ciudadanos a lo largo de la vida, o garantía de prestaciones elevadas en periodo de búsqueda de empleo y de recualificación.

La implementación de estas políticas exige una concienciación tanto de los jefes de Estado y de Gobierno europeos como de los organismos internacionales sobre la ineficacia económica y el coste social de las políticas de austeridad, y de la necesidad de compaginar una gestión rigurosa de las cuentas públicas con unas políticas de reactivación. La llegada a la presidencia de la República francesa del socialista François Hollande y la adhesión de varios gobiernos conservadores a sus tesis sobre la necesidad de fomentar el crecimiento económico y la creación de empleo, están modificando poco a poco la naturaleza del debate político, tanto dentro como fuera de Europa, y la relación de fuerzas en el seno de la Unión europea, sabiendo que Europa constituye el nivel de acción pertinente para implementar las políticas de inversión social.

En efecto, ante el poder creciente de los mercados financieros y de las agencias de notación, por una parte, la escasa eficacia de los políticas estatales y la rápida pérdida de autonomía de los gobiernos nacionales, por otra parte, y el fortalecimiento tanto de la Unión europea, del Fondo Monetario Internacional como del Banco Central Europeo, por último, es indispensable proceder a una mayor integración europea. Dicha integración supone una unión bancaria, con un supervisor y un depósito de garantía europeos; una armonización fiscal; una mutualización de la deuda a través de la creación de eurobonos; una política de inversión europea en sectores de futuro; la modificación del estatus del Banco Central Europeo para que sus misiones no se reduzcan a la lucha contra la inflación; o la transformación de la gobernanza europea mediante la constitución de un verdadero gobierno económico.

\section{Referencias bibliográficas}

Aghion, P. 2010. L'excellence universitaire: leçons des expériences internationales. Paris: Ministère de l'Education supérieure et de la Recherche.

Aghion, P. et al. 2010. "The Governance and Performance of Research Universities: Evidence from Europe and the US", Economic Policy, vol.61, pp.7-59.

Aghion, P. y Cohen, E. 2004. Education et croissance. Paris: Conseil d'analyse économique.

Baudelot, C y Establet, R 2009. L'élitisme républicain. Paris: Seuil. 
Blanpain, N. y Chardon, O. 2010. "Projection de population à I'horizon 2060: un tiers de la population âgé de plus de 60 ans", INSEE Première, ${ }^{\circ} 1320$.

Bluestone, B. y Harrison, B. 1982. The Desindustrialization of America. New York: Basic Books.

Castel, R 1999. Les métamorphoses de la question sociale. Paris: Folio essais.

Cohen, D 2006. Trois leçons sur la société postindustrielle. Paris: Seuil.

Del Pino, E. 2009. "Un marco conceptual para estudiar el cambio del Estado de bienestar y las políticas sociales", en Moreno, L. (ed.): Reformas de las políticas de bienestar en España. Madrid: Siglo XXI, pp.35-66.

Esping-Andersen, G. (ed.), 1996. Welfare States in Transition. London: Sage.

Esping-Andersen, G. 1999. Social Foundations of Postindustrial Societies. Oxford: Oxford University Press.

Esping-Andersen, G 2002. Why We Need a New Welfare State? Oxford: Oxford University Press.

Esping-Andersen, G y Palier, B 2008. Trois leçons sur l'Etat-providence. Paris: Seuil.

Flora, P. (ed.), 1986. Growth to Limits. The European Welfare States since World War II. Berlin: de Gruyter.

Fourastié, J 1979. Les Trente Glorieuses ou la révolution invisible de 1946 a 1975. Paris: Fayard.

Garrett, G. 1998. Partisan Politics in the Global Economy. Cambridge: Cambridge University Press.

Gillion, C., Turner, J., Bailey, C. y Latulippe, D. 2000. Social Security Pensions: Development and Reform. Genève: BIT.

Hayek, F. 1998. Los fundamentos de la libertad. Madrid: Unión Editorial.

Herzog, P. 2004. "Quand I'Europe s'ouvre aux services publics", L'Option, n²0.

Kautto, M., Heikkilä, M., Hvinden, B., Marklund, S y Ploug, N. (eds.), 1999. Nordic Social Policy. London: Routledge.

Keohane, R. y Milner, H. (ed.), 1996. Internationalization and Domestic Politics. New York: Cambridge University Press.

Manow, P. 2001. "Comparative Institutional Advantages of Welfare State Regimes and New Coalitions in Welfare State Reform," in Pierson, P., The New Politics of the Welfare State. Oxford: Oxford University Press, pp.146-163.

Merrien, F-X., Parchet, R. y Kernen, A. 2005. L'Etat social. Une perspective internationale. Paris: Armand Colin.

Palier, B (ed.), 2010. A Long Good Bye to Bismarck? The Politics of Welfare Reforms in Continental Europe. Amsterdam: Amsterdam University Press.

Pierson, P (ed.), 2001. The New Politics of the Welfare State. Oxford: Oxford University Press.

Ramos, J-A. y Del Pino, E. 2009. “Un análisis del cambio en el sistema de pensiones en España", en Moreno, L. (ed.): Reformas de las políticas de bienestar en España. Madrid: Siglo XXI, pp.67-100.

Sassen, S. 1996. Losing control: Sovereignty in an Age of Globalization. New York: Columbia University Press. 
Urteaga, E. 2007. "El modelo escandinavo y su transposición", Revista de servicios sociales y política social, n79, pp.127-148.

Urteaga, E. 2009a. "Las políticas de lucha contra la pobreza y la exclusión social en Francia", Zerbitzuan, n45, pp.7-21.

Urteaga, E. 2009b. "Fundamentos y políticas sociales en Francia", Portularia, vol.9, n², pp.75-95.

Urteaga, E. 2009c. "La política de la familia y de la pequeña infancia", Humanismo y trabajo social, n8, pp.177-199.

Urteaga, E. 2011. "Las rentas mínimas en Francia", Papers, n93 (3), pp.959-983.

Urteaga, E: 2012. "El quinto pilar de la protección social: la política de dependencia en Francia", Cuadernos de Trabajo Social, n²5, pp.221-235.

Von Mises, L. 1975. Liberalismo. Madrid: Unión Editorial.

Wood, A. 1994. North-South Trade. Employment and Inequality: Changing Fortunes in a Skill-Driven World. Oxford: Oxford Clarendon Press. 


\section{comunitania}

REVISTA INTERNACIONAL DE TRABAJO SOCIAL Y CIENCIAS SOCIALES

REVISTA INTERNACIONAL DE TRABAJO SOCIAL Y CIENCIAS SOCIALES
INTERNATIONAL. JOURNAL OF SOCIAL WORK AND SOCIAL SCIENCES

\section{ARTICULOS/ARTICLES}

Estado de bienestar e inversión social en Europa / Welfare State and Social Invesment in Europe

Eguzki Urteaga

Reflexiones sobre el Espacio Público y Participación en las Políticas de Vivienda en Santiago de Chile. Hacia una Práctica Comunitaria / Reflections, on public space and participation in public housinng policies in Santiago de Chile. Towards a community practice

Rodrigo Andrés Mardones

El déficit de transversalidad de género en la Unión Europea / Gender transversality deficit in the European Union

Ángeles Martínez Boyé

Págs 37-60

La construcción de la masculinidad y su relación con la violencia de género / The development of masculinity and relationship to gender violence

$\mathrm{M}^{a}$ Inmaculada López Núñez

Elementos del proceso de mediación que favorecen a su implementación como política social / Elements of the mediation process that benefits implementation as social policy

Paris Alejandro Cabello Tijerina

La mediación escolar y las habilidades sociales en los estudiantes de educación secundaría. Un estudio en institutos de la región de Murcia / School mediation and social skills students in secondary education. A study in institutes of Murcia

María Paz García-Longoria Serrano y Reyna Lizeth Vázquez Gutiérrez

La actuación de los jueces estatales como árbitros privados: un problema de orden público /

State Judge's acting as private arbitrators: a public policy problem

Arnulfo Sánchez García

Págs 137-151

Calidad en los servicios de bienestar en un contexto de crisis económica internacional / Quality in

welfare services in a context of international economic crisis

Eva Sotomayor Morales, Yolanda De la Fuente Robles, Marta García Domingo,

$\mathrm{M}^{\mathrm{a}}$ Luisa Grande Gascón y Tomás Alberich Nistal

Págs 153-179

Contexto de la equidad en los servicios de salud / Context of equity in health services in Mexico

Karla Sáenz López y Delia Téllez Castilla

Los Servicios Sociales en España, puestos a prueba por las personas inmigrantes / Social Services

in Spain, tested by inmigrants

Antonio Gutiérrez Resa

\section{RESEẼAS/REVIEWS}

Rubén Darío Torres Kumbrián. Trabajo Social con Comunidades y Mujeres Musulmanas:

"Diagnóstico de las discriminaciones múltiples" / Social Work with Communities and Muslim women (por Ángeles Martínez Boyé).

Págs 229-234

Manuel Herrera Gómez y José Daniel Barquero Cabrero. Redes sociales: de metáfora a paradigma /

Social Networks: from metaphor to paradigm (por Salvador Gómez García)......

Djamil Tony Kahale Carrillo, Protección a las personas en situación de dependencia / Protection of persons in situations of dependency (por Sara Pérez Martínez). 